\title{
Orbital shadowing property on chain transitive sets for generic diffeomorphisms
}

\author{
Manseob Lee \\ Department of Mathematics, \\ Mokwon University, \\ Daejeon, 302-729, Korea \\ email: Imsds@mokwon.ac.kr
}

\begin{abstract}
Let $f: M \rightarrow M$ be a diffeomorphism on a closed smooth $n(\geq 2)$ dimensional manifold $M$. We show that $C^{1}$ generically, if a diffeomorphism $f$ has the orbital shadowing property on locally maximal chain transitive sets which admits a dominated splitting then it is hyperbolic.
\end{abstract}

\section{Introduction}

Let $M$ be a closed smooth $\mathfrak{n}(n \geq 2)$-dimensional Riemannian manifold, and let $\operatorname{Diff}(M)$ be the space of diffeomorphisms of $M$ endowed with the $C^{1}$-topology. Denote by $\mathrm{d}$ the distance on $M$ induced from a Riemannian metric $\|\cdot\|$ on the tangent bundle TM. For any $\delta>0$, a sequence $\left\{x_{i}\right\}_{i \in \mathbb{Z}}$ is called a $\delta$-pseudo orbit of $f$ if $d\left(f\left(x_{i}\right), x_{i+1}\right)<\delta$ for all $i \in \mathbb{Z}$. Let $\Lambda$ be a closed $f$-invariant set. We say that $f$ has the shadowing property on $\Lambda$ if for any $\epsilon>0$ there is $\delta>0$ such that for any $\delta$-pseudo orbit $\left\{x_{i}\right\}_{i \in \mathbb{Z}} \subset \Lambda$ there is $y \in M$ such that $d\left(f^{i}(y), x_{i}\right)<\epsilon$ for all $i \in \mathbb{Z}$. If $\Lambda=M$ then we say that $f$ has the shadowing property. The shadowing property is very useful notion to investigate for hyperbolic structure. In fact, Robinson[22] and Sakai[24] proved that a diffeomorphism $f$ has the $\mathrm{C}^{1}$ robustly shadowing property if and only if it is structurally stable

2010 Mathematics Subject Classification: Primary 37C50; Secondary 34D10 Key words and phrases: shadowing; orbital shadowing, chain transitive set, homoclinic class, chain recurrence class, hyperbolic, generic 
diffeomorphisms. Here, we say that $f$ has the $C^{1}$ robustly shadowing property if there is a $C^{1}$ neighborhood $\mathcal{U}(\mathrm{f})$ of $\mathrm{f}$ such that for any $\mathrm{g} \in \mathcal{U}(\mathrm{f}), \mathrm{g}$ has the shadowing property. From the property, a general shadowing property was introduced by [20] which is called the orbital shadowing property. For the orbital shadowing property, many results published by the various view points (see $[10,13,14,15,16,17,19]$ ). We say that $f$ has the orbital shadowing property on $\Lambda$ if for any $\epsilon>0$ there is $\delta>0$ such that for any $\delta$ pseudo orbit $\xi=\left\{x_{i}\right\}_{\in \mathbb{Z}} \subset \Lambda$ such that there is a point $y \in M$ such that

$$
\operatorname{Orb}(y) \subset \mathrm{B}_{\epsilon}(\xi) \text { and } \xi \subset \mathrm{B}_{\epsilon}(\mathrm{Orb}(\mathrm{y})) \text {. }
$$

If $\Lambda=M$ then we say that $f$ has the orbital shadowing property. Let $\Lambda$ be a closed $f$-invariant set. We say that $\Lambda$ is hyperbolic if the tangent bundle $\mathrm{T}_{\mathcal{\Lambda}} \mathrm{M}$ has a Df-invariant splitting $\mathrm{E}^{\mathrm{s}} \oplus \mathrm{E}^{\mathfrak{u}}$ and there exist constants $\mathrm{C}>0$ and $0<\lambda<1$ such that

$$
\left\|\left.D_{x} f^{n}\right|_{E_{x}^{s}}\right\| \leq C \lambda^{n} \text { and }\left\|\left.D_{x} f^{-n}\right|_{E_{x}^{u}}\right\| \leq C \lambda^{n}
$$

for all $x \in \Lambda$ and $n \geq 0$. If $\Lambda=M$ then we say that $f$ is Anosov.

Pilyugin et al [20] proved that if a diffeomorphism $f$ has the $C^{1}$ robustly orbital shadowing property then it is structurally stable diffeomorphisms. Lee and Lee $[10]$ proved that a volume preserving diffeomorphism $f$ has the $C^{1}$ robustly orbital shadowing property then it is Anosov. Moreover, we can find similar results $[12,13,14,15]$. We say that the set $\Lambda$ is transitive if there is a point $x \in \Lambda$ such that $\omega(x)=\Lambda$, where $\omega(x)$ is the omega limit set of $x$. An invariant closed set $\mathcal{C}$ is called a chain transitive if for any $\delta>0$ and $x, y \in \mathcal{C}$, there is $\delta$-pseudo orbit $\left\{x_{i}\right\}_{i=0}^{n}(n \geq 1) \subset \mathcal{C}$ such that $x_{0}=x$ and $x_{n}=y$. It is clear that the transitive set $\Lambda$ is the chain transitive set $\mathcal{C}$, but the converse is not true. We say that $\Lambda$ is locally maximal if there is a neighborhood $U$ of $\Lambda$ such that $\Lambda=\bigcap_{n \in \mathbb{Z}} f^{n}(U)$. For the relation between chain transitive sets and $\mathrm{C}^{1}$ robustly shadowing theories, In [16], Lee proved that if a robustly chain transitive set with orbital shadowing then it is hyperbolic. We say that $f$ has the $C^{1}$ stably shadowing property on $\Lambda$ if there are a $C^{1}$ neighborhood $\mathcal{U}(f)$ of $f$ and a neighborhood $U$ of $\Lambda$ such that for any $g \in \mathcal{U}(f), g$ has the shadowing property on $\Lambda_{g}(U)$, where $\Lambda_{g}(U)$ is the continuation of $\Lambda$. For $f \in \operatorname{Diff}(M)$, we say that a compact $\mathrm{f}$-invariant set $\Lambda$ admits a dominated splitting if the tangent bundle $T_{\Lambda} M$ has a continuous Df-invariant splitting $E \oplus F$ and there exist $C>0,0<\lambda<1$ such that for all $x \in \Lambda$ and $n \geq 0$, we have

$$
\left\|\left.D f^{n}\right|_{E(x)}\right\| \cdot\left\|\left.D f^{-n}\right|_{F\left(f^{n}(x)\right)}\right\| \leq C \lambda^{n} .
$$


In [18], Lee proved that if a diffeomorphism $f$ has the $C^{1}$ stably shadowing property on chain transitive set $\mathcal{C}$ then it admits a dominated splitting. Sakai [23] proved that a diffeomorphism $f$ has the $C^{1}$ stably shadowing property on chain transitive set $\mathcal{C}$ then it is hyperbolic.

We say that a subset $\mathcal{G} \subset \operatorname{Diff}(M)$ is residual if $\mathcal{G}$ contains the intersection of a countable family of open and dense subsets of $\operatorname{Diff}(M)$; in this case $\mathcal{G}$ is dense in $\operatorname{Diff}(M)$. A property " $P$ " is said to be $\left(C^{1}\right)$-generic if " $P$ " holds for all diffeomorphisms which belong to some residual subset of Diff $(M)$. For $C^{1}$ generic differeomorphism $\mathrm{f}$, Abdenur and Díaz [3] suggested the problem : if a $\mathrm{C}^{1}$ generic diffeomorphism $\mathrm{f}$ has the shadowing property then is it hyperbolic?

Unfortunately, this question still is open. For the problem, there are partial results $[4,9,11]$. Ahn et al [4] proved that for $\mathrm{C}^{1}$ generic diffeomorphism $\mathrm{f}$, if $f$ has the shadowing property on a locally maximal homoclinic class then it is hyperbolic. Lee and Wen [11] proved that for $C^{1}$ generic diffeomorphism $f$, if $f$ has the shadowing property on a locally maximal chain transitive set then it is hyperbolic. Very recently, Lee and Lee [9] proved that for $C^{1}$ generic diffeomorphism $f$, if $f$ has the shadowing property on chain recurrence classes then it is hyperbolic. From the results, we study the orbital shadowing property for $\mathrm{C}^{1}$ generic diffeomorphisms. The following is the main theorem of the paper.

Theorem A For $\mathrm{C}^{1}$ generic $\mathrm{f}$, if $\mathrm{f}$ has the orbital shadowing property on a locally maximal $\mathcal{C}$ which admits a dominated splitting $\mathrm{E} \oplus \mathrm{F}$ then it is hyperbolic.

\section{Proof of Theorem A}

Let $M$ be as before, and let $f \in \operatorname{Diff}(M)$. A periodic point for $f$ is a point $p \in M$ such that $f^{\pi(p)}(p)=p$, where $\pi(p)$ is the minimum period of $p$. Denote by $P(f)$ the set of all periodic points of $f$. Let $p$ be a hyperbolic periodic point of $f$. A point $x \in M$ is called chain recurrent if for any $\delta>0$, there is a finite $\delta$-pseudo orbit $\left\{x_{i}\right\}_{i=0}^{n}(n \geq 1)$ such that $x_{0}=x$ and $x_{n}=x$. Denote by $\mathcal{C R}(f)$ the set of all chain recurrent points of $f$. We define a relation $\leadsto$ on $\mathcal{C R}(f)$ by $x \leftrightarrow y$ if for any $\delta>0$, there is a finite $\delta$ pseudo orbit $\left\{x_{i}\right\}_{i=0}^{n}$ such that $x_{0}=x$ and $x_{n}=y$ and a finite $\delta$ pseudo orbit $\left\{w_{i}\right\}_{i=0}^{n}$ such that $w_{0}=y$ and $w_{n}=x$. Then we know that the relation $\leadsto$ is an equivalence relation on $\mathcal{C R}(f)$. the equivalence classes are called the chain recurrence classes of $f$, denote by $C_{f}$. Note that if the class $C_{f}$ has a hyperbolic periodic point $p$ then we denote as $C(p, f)$.

It is well known that if $p$ is a hyperbolic periodic point of $f$ with period $k$ 
then the sets

$$
\begin{aligned}
& W^{s}(p)=\left\{x \in M: f^{k n}(x) \rightarrow p \text { as } n \rightarrow \infty\right\} \text { and } \\
& W^{\mathfrak{u}}(p)=\left\{x \in M: f^{-k n}(x) \rightarrow p \text { as } n \rightarrow \infty\right\}
\end{aligned}
$$

are $\mathrm{C}^{1}$-injectively immersed submanifolds of $\mathrm{M}$. The homoclinic class of a hyperbolic periodic point $p$ is the closure of the transverse intersection of the $W^{s}(p)$ and $W^{\mathfrak{u}}(p)$, and it is denoted by $H(p, f)$. It is clear that $H(p, f)$ is compact, transitive and invariant sets. Let $q$ be a hyperbolic periodic point of $\mathrm{f}$. We say that $\mathrm{p}$ and $\mathrm{q}$ are homoclinically related, and write $\mathrm{p} \sim \mathrm{q}$ if

$$
W^{s}(p) \pitchfork W^{\mathfrak{u}}(q) \neq \emptyset \text { and } W^{\mathfrak{u}}(p) \pitchfork W^{s}(q) \neq \emptyset \text {. }
$$

It is clear that if $p \sim q$ then $\operatorname{index}(p)=\operatorname{index}(q)$, that is, $\operatorname{dim}^{s}(p)=$ $\operatorname{dim} W^{s}(q)$. By the Smale's transverse homoclinic point theorem, $H_{f}(p)$ coincides with the closure of the set of hyperbolic periodic points $q$ of $f$ such that $p \sim q$. Note that if $p$ is a hyperbolic periodic point of $f$ then there is a neighborhood $\mathcal{U}$ of $p$ and a $C^{1}$-neighborhood $\mathcal{U}(f)$ of $f$ such that for any $g \in \mathcal{U}(f)$ there exists a unique hyperbolic periodic point $p_{g}$ of $g$ in $U$ with the same period as $p$ and $\operatorname{index}\left(p_{g}\right)=\operatorname{index}(p)$. Such a point $p_{g}$ is called the continuation of $p=p_{f}$. The following are results for $C^{1}$ generic diffeomorphisms (see [2]).

Lemma 1 There is a residual set $\mathcal{G} \subset \operatorname{Diff}(M)$ such that if $\mathrm{f} \in \mathcal{G}$,

(a) $\mathrm{H}(p, f)=C(p, f)$, for some hyperbolic periodic point $\mathrm{p}$ (see [5]).

(b) A compact $\mathrm{f}$-invariant set $\mathcal{C}$ is chain transitive if and only if $\mathcal{C}$ is the Hausdorff limit of a sequence of periodic orbits of $\mathrm{f}$ (see [8]).

(c) A locally maximal transitive set $\Lambda$ is a locally maximal $\mathrm{H}(\mathrm{p}, \mathrm{f})$ for some periodic point $\mathrm{p} \in \Lambda$ (see [1]).

(d) $H(p, f)=\overline{W^{s}(p)} \cap \overline{W^{u}(p)}$ (see [7]).

Remark 1 Applying Pugh's closing lemma, we know that any transitive set $\Lambda$ of a $\mathrm{C}^{1}$-generic diffeomorphism $\mathrm{f}$ is the Hausdorff limit of a sequence of periodic orbits $\operatorname{Orb}_{\mathrm{f}}\left(\mathrm{p}_{\mathrm{n}}\right)$ of $\mathrm{f}$, that is, $\lim _{\mathrm{n} \rightarrow \infty} \operatorname{Orb}_{\mathrm{f}}\left(\mathrm{p}_{\mathrm{n}}\right)=\Lambda$. By Lemma 1 (b) and (c), a chain transitive set $\mathcal{C}$ is a transitive set $\Lambda$ and so, a locally maximal chain transitive set $\mathcal{C}=\mathrm{H}(\mathrm{p}, \mathrm{f})$ for some periodic point $\mathrm{p}$.

Let $\Lambda$ be a closed $f$-invariant set. We say that $\Lambda$ is Lyapunov stable for $f$ if for any open neighborhood $\mathrm{U}$ of $\Lambda$ there is a neighborhood $\mathrm{V} \subset \mathrm{U}$ such that 
$f^{j}(V) \subset U$ for all $j \in \mathbb{N}$. We say that the closed set is bi-Lyapunov stable if it is Lyapunov stable for $f$ and $f^{-1}$. Potrie [21, Theorem 1.1] proved that $C^{1}$ generically, if a homoclinic class $\mathrm{H}(p, f)$ is a bi-Lyapunov stable then it admits a dominated splitting.

A diffeomorphism $f$ has a heterodimensiional cycle associated with the hyperbolic periodic points $p$ and $q$ of $f$ if (i) the indice of the points $p$ and $q$ are different, and (ii) the stable manifold of $p$ meets the unstable manifold of $q$ and the same holds for the stable manifold of $p$ and the unstable manifold of $\mathrm{q}$ (see [6]). We say that $\mathrm{f}$ has $\mathrm{C}^{1}$ robustly heterodimensional cycle if $\mathrm{f}$ has a heterodiemnsional cycle associated with the hyperbolic periodic points $p$ and $q$ of $f$ and there is a $C^{1}$ neighborhood $\mathcal{U}(f)$ of $f$ such that for any $g \in \mathcal{U}(f), g$ has a heterodimensional cycle associated with the hyperbolic periodic points $p_{g}$ and $q_{g}$, where $p_{g}$ and $q_{g}$ are the continuations of $p$ and $q$ for $g$.

Lemma 2 [6, Corollary 1.15] There is a residual set $\mathcal{T} \subset \operatorname{Diff}(M)$ such that for any $f \in \mathcal{T}$ and every locally maximal chain recurrence class $\mathrm{C}_{\mathrm{f}}$ of $f$ there are two possibilities: either $\mathrm{C}_{\mathrm{f}}$ is hyperbolic or it has a robustly heterodimensional cycle.

Lemma 3 Let $\mathrm{C}(\mathrm{p}, \mathrm{f})$ admits a dominated splitting $\mathrm{E} \oplus \mathrm{F}$ and let $\mathrm{C}(\mathrm{p}, \mathrm{f})$ be locally maximal. If a chain recurrence class $\mathrm{C}(\mathrm{p}, \mathrm{f})$ has heterodimensional cycle then $\mathrm{f}$ does not have the orbital shadowing property on $\mathrm{C}(\mathrm{p}, \mathrm{f})$.

Proof. Suppose, by contradiction, that $f$ has the orbital shadowing property on $C(p, f)$. Since $C(p, f)$ has heterodimensional cycle, there is $q$ a hyperbolic periodic point in $C(p, f)$ such that index $(p) \neq \operatorname{index}(q)$. Then we take $x, y \in$ $C(p, f)$ such that $x \in W^{s}(p) \cap W^{\mathfrak{u}}(q)$ and $y \in W^{\mathfrak{u}}(p) \cap W^{s}(q)$. By assumption, $x, y$ are not transverse intersection points. Since $q$ is hyperbolic, $T_{q} M=E_{q}^{s} \oplus$ $E_{q}^{u}$. Choose $\alpha>0$ sufficiently small such that $W_{\alpha / 4}^{s}(q)=\exp _{q}\left(E^{s}(\alpha / 4)\right)$ and $W_{\alpha / 4}^{\mathfrak{u}}(q)=\exp _{\mathbf{q}}\left(E^{\mathfrak{u}}(\alpha / 4)\right)$. Then we may assume that $y \in W_{\alpha / 4}^{\mathrm{s}}(\mathbf{q})$ and $x \in$ $W_{\alpha / 4}^{\mathfrak{u}}(q)$. Since $y \in W^{\mathfrak{u}}(p)$, there is $\eta>0$ such that $y \in B_{\eta}(y) \cap W^{\mathfrak{u}}(p)$. Take a small arc $\mathcal{J}_{y} \subset B_{\eta}(y) \cap W^{\mathfrak{u}}(p)$ such that $T_{y} \mathcal{J}_{y}=T_{y} W^{\mathfrak{u}}(p)$. Since $C(p, f)$ admits a dominated splitting $E \oplus F$, we have $T_{y} \mathcal{J}_{y}=F_{y}=T_{y} W^{u}(p), F_{q} \subset E_{q}^{u}$ and $E_{q}^{s} \subset E_{q}$. Put $E^{\mathfrak{u}, 1}=E_{q} \oplus E_{q}^{u}$ and $E^{u, 2}=F_{q}$. Then $E_{q}^{u}=E_{q}^{u, 1} \oplus E_{q}^{u, 2}$, and $W_{\alpha / 4}^{u, 1}(q)=\exp _{q}\left(E_{q}^{u, 1}(\alpha / 4)\right), W_{\alpha / 4}^{u, 2}(q)=\exp _{q}\left(E_{q}^{u, 2}(\alpha / 4)\right)$.

Let $\mathrm{P}^{\mathrm{u}}: \mathrm{B}_{\alpha / 4}(\mathrm{q}) \rightarrow \mathrm{E}_{\mathrm{q}}^{\mathrm{u}}$ and $\mathrm{P}^{\mathrm{s}}: \mathrm{B}_{\alpha / 4}(\mathrm{q}) \rightarrow \mathrm{E}_{\mathrm{q}}^{\mathrm{s}}$ be the projections parallel to $E_{q}^{s}$ and $E_{q}^{u}$, respectively. Then $P^{u}\left(f^{n}\left(\mathcal{J}_{y}\right)\right) \cap B_{\alpha / 4}(q) \rightarrow W_{\alpha / 4}^{u, 1}(q)$ and $\mathrm{P}^{\mathrm{s}}\left(\mathrm{f}^{\mathrm{n}}\left(\mathcal{J}_{\mathrm{y}}\right)\right) \cap \mathrm{B}_{\alpha / 4}(\mathrm{q}) \rightarrow \mathrm{q}$ as $\mathrm{n} \rightarrow \infty$. 
Take $\epsilon=\min \left\{\alpha / 4, \eta, d\left(x, W_{\alpha / 4}^{u, 1}(p)\right) / 2\right\}$, and let $0<\delta<\epsilon$ be the number of the orbital shadowing property. Since $y \in W^{\mathcal{s}}(q) \cap W^{\mathfrak{u}}(p)$ and $x \in$ $W^{\mathfrak{u}}(q) \cap W^{s}(p)$, there are $i_{1}>0$ and $i_{2}>0$ such that (i) $d\left(f^{i_{1}}(y), f^{-i_{1}}(x)\right)<\delta$ and $d\left(f^{-i_{2}}(y), f^{i_{2}}(x)\right)<\delta$, (ii) $\max \left\{d_{H}\left(P^{s}\left(f^{i_{1}+j}\left(\mathcal{J}_{y}\right), q\right), d_{H}\left(P^{u}\left(f^{i_{1}+j}\left(\mathcal{J}_{y}\right)\right) \cap\right.\right.\right.$ $\left.\left.B_{\alpha / 4}(q), W_{\alpha / 4}^{u, 1}(q)\right)\right\}<\epsilon$ for all $j \geq 0$, where $d_{H}$ is the Hausdorff metric. Then we have a $\delta$-pseudo orbit as follows:

$$
\begin{aligned}
\xi= & \left\{y, f(y), \ldots, f^{i_{1}-1}(y), f^{-i_{1}}(x), \ldots, f^{-1}(x),\right. \\
& \left.x, f(x), \ldots, f^{i_{2}-1}(x), f^{-i_{2}}(y), \ldots, f^{-1}(y), y\right\} \subset C(p, f) .
\end{aligned}
$$

Since $f$ has the orbital shadowing property on $C(p, f)$ and $C(p, f)$ is locally maximal, there is a point $w \in C(p, f)$ such that

$$
\operatorname{Orb}(w) \subset \mathrm{B}_{\epsilon}(\xi) \text { and } \xi \subset \mathrm{B}_{\epsilon}(\mathrm{Orb}(w)) .
$$

First, we assume that there is $k>0$ such that $f^{k}(w) \in \mathcal{J}_{y} \backslash\{y\}$. Then if $f^{k}(w) \in P^{u}\left(f^{n}\left(\mathcal{J}_{y}\right)\right)$ then since $\mathrm{P}^{\mathfrak{u}}\left(\mathrm{f}^{\mathrm{n}}\left(\mathcal{J}_{y}\right)\right) \rightarrow \mathrm{W}^{\mathrm{u}, 1}(\mathrm{q})(\mathrm{n} \rightarrow \infty), \mathrm{f}^{\mathrm{k}+\mathrm{n}}(w) \rightarrow$ $W^{\mathfrak{u}, 1}(q)$ as $n \rightarrow \infty$. Thus there is $j>0$ such that $d\left(f^{k+j}(w), f^{j}(y)\right)>8 \epsilon$ and so, $\mathrm{d}\left(\mathrm{f}^{\mathrm{k}+\mathrm{j}}(w), \mathrm{q}\right)>2 \epsilon$ which is a contradiction. If $\mathrm{f}^{\mathrm{k}}(w) \notin \mathrm{P}^{\mathrm{u}}\left(\mathcal{J}_{\mathrm{y}}\right)$ then by $\lambda$-lemma, $f^{n}\left(\mathcal{J}_{y}\right) \rightarrow W^{\mathfrak{u}}(q)$ as $n \rightarrow \infty$. Then there is $l>0$ such that $d\left(f^{k+l}(w), q\right)>4 \epsilon$. Since $x \in W^{\mathfrak{u}}(q)$, there is $m>0$ such that $d\left(f^{-m}(x), q\right)<$ $\epsilon$ for some $m<i_{2}$. Then we know that $f^{k+l}(w) \notin B_{\epsilon}(\xi)$ which is a contradiction by the orbital shadowing property on $\mathrm{C}(\mathrm{p}, \mathrm{f})$. Then for all $i \in \mathbb{Z}, \mathrm{f}^{\mathrm{i}}(\mathcal{w}) \notin$ $\mathcal{J}_{y} \backslash\{y\}$.

We assume that there is $k>0$ such that $f^{k}(w)=y$. Since $y \in W^{s}(q) \cap W^{\mathfrak{u}}(p)$ and $x \in W^{\mathfrak{u}}(q) \cap W^{s}(p)$, we know $\operatorname{Orb}(x) \cap \operatorname{Orb}(y)=\emptyset$. Then we have $\xi \not \subset \mathrm{B}_{\epsilon}(\mathrm{Orb}(w))$ which is a contradiction by the orbital shadowing property on $\mathrm{C}(\mathrm{p}, \mathrm{f})$. Thus we know $\operatorname{Orb}(w) \cap \mathcal{J}_{y}=\emptyset$.

Finally, we assume that there is $k>0$ such that $f^{k}(w) \in B_{\eta}(y) \backslash \mathcal{J}_{y}$. Then for all $z \in B_{\eta}(y) \backslash \mathcal{J}_{y}$, there is $k>0$ such that $d\left(f^{-k}(x), f^{k}(z)\right)>2 \epsilon$ since $x \in W^{\mathfrak{u}}(q)$ and $q$ is hyperbolic saddle. Then we have $\xi \not \subset B_{\epsilon}(\operatorname{Orb}(w))$ which is a contradiction by the orbital shadowing property on $C(p, f)$. Consequently, if a locally maximal chain recurrence class $C(p, f)$ admits a dominated splitting and $f$ has the orbital shadowing property on $C(p, f)$ then it does not the heterodimensional cycle.

Proof of Theorem A. Let $f \in \mathcal{G} \cap \mathcal{T}$ and let $f$ has the orbital shadowing property on a locally maximal chain transitive set $\mathcal{C}$. Since $f \in \mathcal{G}$, by Remark $1 \mathcal{C}=\mathrm{C}(\mathrm{p}, \mathrm{f})$ for some hyperbolic periodic point $\mathrm{p}$. Since chain transitive set $\mathcal{C}$ admits a dominated splitting $E \oplus F$, and $f$ has the orbital shadowing property 
on a locally maximal chain transitive $\mathcal{C}$, by Lemma $3, \mathcal{C}=\mathrm{C}(\mathrm{p}, \mathrm{f})$ does not have the heterodimensional cycles. It is clear that a locally maximal $C(p, f)$ does not have the robustly hetrodimensional cycle. Thus by Lemma 2, a locally maximal chain transitive set $\mathcal{C}$ is hyperbolic.

In Abdenur et al [2] the authors proved that every locally maximal homoclinic class with a non-empty interior is the whole space.

Corollary 1 Let $\mathrm{f}: \mathrm{M} \rightarrow \mathrm{M}$ be a diffeomorphism with $\operatorname{dim} \mathrm{M}=3$. For $\mathrm{C}^{1}$ generic $\mathrm{f}$, if $\mathrm{f}$ has the orbital shadowing property on a locally maximal chain transitive set $\mathcal{C}$ which admits a dominated splitting $\mathrm{E} \oplus \mathrm{F}$ then it is Anosov.

The following was proved in [21, Proposition 1.2] which means that a homoclinic class admits a codimension one dominated splitting then it has a non-empty interior.

Lemma 4 There is a residual set $\mathcal{R} \subset \operatorname{Diff}\left(M^{3}\right)$ such that for any $\mathrm{f} \in \mathcal{R}$, if a homoclinic class $\mathrm{H}$ admits a codimension one dominated splitting then it has non-empty interior.

Proof of Corollary 1. Let $f \in \mathcal{G} \cap \mathcal{T} \cap \mathcal{R}$ and let $\mathrm{f}$ has the orbital shadowing property on a locally maximal chain transitive set $\mathcal{C}$ which admits a dominated splitting $E \oplus \mathrm{F}$. By Remark 1, a locally maximal chain transitive $\mathcal{C}=\mathrm{H}(\mathrm{p}, \mathrm{f})$. Since $\mathrm{f} \in \mathcal{R}$, by Lemma 4 , a hoomoclinic class $\mathrm{H}(\mathrm{p}, \mathrm{f})$ has nonempty interior. Since $H(p, f)$ is locally maximal, by $[2$, Thereom 3$], H(p, f)=M$. Since $f$ has the orbital shadowing property on a locally maximal chain transitive set $\mathcal{C}$ which admits a dominated splitting $\mathrm{E} \oplus \mathrm{F}$, by Theorem A, it is hyperbolic, and so it is Anosov.

\section{Acknowledgement}

The author would like to thank the referee for valuable help in improving the presentation of this article.

\section{References}

[1] F. Abdenur, C. Bonatti and S. Crovisier, Nonuniform hyperbolicty for $C^{1}$-generic diffeomorphisms, Isral J. Math., 183 (2011), 1-60.

[2] F. Abdenur, C. Bonatti and L. J. Díaz, Non-wandering sets with non empty interior, Noinearity, 17 (2004), 175-191. 
[3] F. Abdenur and L. J. Díaz, Pseudo-orbit shadowing in the $\mathrm{C}^{1}$-topology, Discrete Contin. Dyn. Syst., 17 (2) (2007), 223-245.

[4] J. Ahn, K. Lee and M. Lee, Homoclinic classes with shadowing, J. Inequal. Appl., 2012:97 (2012), 1-6.

[5] C. Bonatti and S. Crovisier, Récurrence et généricité, Invent. Math., 158 (2004), 33-104.

[6] C. Bonatti and L. J. Díaz, Robust heterodimensional cycles and $\mathrm{C}^{1}$ generic dynamics, J. Inst. Math. Jessieu, 7 (2008), 469-525.

[7] C. Carballo, C. A. Morales and M. J. Pcaifico, Homoclinic classes for $\mathrm{C}^{1}$ generic vector fields, Ergod. Theorey Dynam. Syst., 23 (2003), 403-415.

[8] S. Crovisier, Periodic orbits and chain transitive sets of $\mathrm{C}^{1}$-diffeomorphisms, Publ. Math. Inst. Hautes Etudes. Sci., 104 (2006), 87-141.

[9] K. Lee and M. Lee, Shadowable chain recurrence classes for generic diffeomorphisms, Taiwan J. Math., 20 (2016), 399-409.

[10] K. Lee and M. Lee, Volume preserving diffeomorphisms with orbital shadowing, J. Inequal. Appl., 2013:18, (2013), 5 pp.

[11] K. Lee and X. Wen, Shadowable chain transitive sets of $C^{1}$ generic diffeomorphisms, Bull. Korean Math. Soc. 49 (2012), 263-270.

[12] M. Lee, Hamiltonian systems with orbital, orbital inverse shadowing, Adv. Difference Equ., 2014:192(2014), 9 pp.

[13] M. Lee, Orbital shadowing property for generic divergence-free vector fields, Chaos Solitons $\&$ Fractals, 54 (2013), 71-75.

[14] M. Lee, Orbital shadowing for $C^{1}$-generic volume-preserving diffeomorphisms, Abstr. Appl. Anal., 2013, Art. ID 693032, 4 pp.

[15] M. Lee, Divergence-free vector fields with orbital shadowing, Adv. Difference Equ, 2013:132, (2013), 6 pp.

[16] M. Lee, Robustly chain transitive sets with orbital shadowing diffeomorphisms, Dyn. Syst., 27 (2012), 507-514.

[17] M. Lee, Chain components with $\mathrm{C}^{1}$-stably orbital shadowing, Adv. Difference Equ., 2013:67 (2013), 12 pp. 
[18] M. Lee, Chain transitive sets with dominated splitting, J. Math. Sci. Adv. Appl., 4 (2010), 201-208.

[19] A. V. Osipov, Nondensity of the orbital shadowing property in $\mathrm{C}^{1}$ topology, (Russian) Algebra i Analiz 22 (2010), no. 2, 127-163; translation in St. Petersburg Math. J., 22 (2) (2011), 267-292.

[20] S. Y. Pilyugin, A. A. Rodionova and K. Sakai, Orbital and weak shadowing properties, Discrete Contin. Dyn. Syst., 9 (2) (2003), 287-308.

[21] R. Potrie, Generic bi-Lyapunov stable homoclinic classes, Nonlinearity, 23 (2010), 1631-1649.

[22] C. Robinson, Stability theorem and hyperbolicity in dynamical systems, Rocky Mountain J. Math., 7 (1977), 425-437.

[23] K. Sakai, Sahdowable chain transitive sets, J. Diff. Eqaut. Appl., 19 (2013), 1601-1618.

[24] K. Sakai, Pseudo orbit tracing property and strong transversality of diffeomorphisms on closed manifolds, Osaka J. Math., 31 (1994), 373-386.

Received: September 18, 2018 